\title{
NOTE ON THE OVERCONVERGENCE OF SEQUENCES OF POLYNOMIALS OF BEST APPROXIMATION*
}

\author{
BY \\ J. L. WALSH
}

1. Introduction. It frequently occurs that the sequence of polynomials (in the complex variable) of best approximation to a function $f(z)$ analytic on a given point set $C$ converges to that function $f(z)$ (or its analytic extension) not merely on the given point set $C$ but also on a larger point set containing $C$ in its interior. This phenomenon may be called overconvergence, and for its occurrence the term "best approximation" may be interpreted in any one of several ways. In a recent paper $\dagger$ the present writer pointed out that many results on overconvergence follow from a single theorem on degree of approximation. The present note is essentially a continuation of that previous paper, and indicates several new ways in which the term "best approximation" may be used so that we still have overconvergence. The principal new results are concerned with (1) approximation on an arbitrary rectifiable Jordan arc, approximation to be measured by a line integral on that arc, and (2) approximation on a more general point set $C$ as measured by integration over the unit circle $\gamma:|w|=1$ when the exterior of $C$ is mapped conformally onto the exterior of $\gamma$; as a further new result we add a single remark ( $(9)$ on the exact regions of uniform convergence of the sequences. The previous paper considered the approximation of harmonic functions by harmonic polynomials as well as approximation of analytic functions by polynomials in the complex variable, but the present note deals only (except in \$9) with the latter topic.

If $C$ is an arbitrary closed limited point set of the $z$-plane whose complement (with respect to the entire plane) is a simply connected region $D$, then we denote by $C_{R}$ the Jordan curve which is the locus $|\phi(z)|=R>1$, where $w=\phi(z)$ maps $D$ conformally onto the exterior of $\gamma:|w|=1$ so that the points at infinity correspond to each other. That is, $C_{R}$ is the transform in the $z$ plane of the circle $|w|=R$.

We shall have occasion to apply the following theorem:

* Presented to the Society, September 11, 1930; received by the editors November 14, 1930.

t These Transactions, vol. 32 (1930), pp. 794-816.

$\ddagger$ For the proof and detailed references to the literature, see Walsh, Münchner Berichte, 1926, pp. 223-229. 
THEOREM I. Let $C$ be an arbitrary closed limited point set of the z-plane whose complement (with respect to the entire plane) is simply connected.

If the function $f(z)$ is analytic on and within $C_{R}$, there exist polynomials $\pi_{n}(z)$ of respective degrees* $n=1,2, \cdots$ such that we have

$$
\left|f(z)-\pi_{n}(z)\right| \leqq \frac{M}{R^{n}}, z \text { on } C, M \text { independent of } n \text { and of } z \text {. }
$$

If there exist polynomials $\pi_{n}(z), n=1,2, \cdots$, such that the inequality

$$
\left|f(z)-\pi_{n}(z)\right| \leqq \frac{M}{R^{n}}
$$

is valid for $z$ on $C$, then the function $f(z)$ is analytic interior to $C_{R}$ and the sequence $\left\{\pi_{n}(z)\right\}$ converges interior to $C_{R}$, uniformly on any closed point set interior to $C_{R}$.

In the previous paper we had frequent occasion to apply Theorem I directly; in the present paper we shall ordinarily use rather the method of proof than the theorem itself.

2. A lemma on polynomials. The following lemma is to be used in our later work. The lemma itself is analogous to one used by Bernstein, $\nmid$ although the present proof is related to the proof of Bernstein's Lemma which was given by M. Riesz. $\ddagger$

LEMMa. If on the rectifiable Jordan arc $C$ we have

$$
\int_{C}\left|P_{n}(z)\right| p|d z| \leqq \rho^{p}, p>1,
$$

where $P_{n}(z)$ is a polynomial of degree $n$, then we have

$$
\left|P_{n}(z)\right| \leqq M_{R} \rho R^{n+1}, \quad \text { zon or within } C_{R},
$$

where $M_{R}$ depends on $R$ but not on $\rho$ or $n$.

Consider the z-plane cut along the curve $C$, and let us distinguish between the two banks of the curve. The new curve may be considered a Jordan curve $\Gamma$, and the function $\phi(z)$ considered above is, if suitably defined on $\Gamma$, continuous and single-valued on the entire cut $z$-plane. The function $P_{n}(z) /[\phi(z)]^{n+1}$ is analytic except on $\Gamma$ and continuous on the entire cut plane and vanishes at infinity. Then we have, for $z$ not on $\Gamma$ (the proof is easy to

\footnotetext{
* By a polynomial of degree $n$ is meant an expression of the form $a_{0} z^{n}+a_{1} 7^{n-1}+\cdots+a_{n}$. $\dagger$ Mémoires, Académie Royale de Belgique, Classe des Sciences, (2), vol. 4 (1912), p. 36. $\ddagger$ Acta Mathematica, vol. 40 (1916), pp. 337-347.
} 
give by considering a variable auxiliary Jordan curve $\Gamma_{n}$ which encloses and approaches $\Gamma$ ) Cauchy's formula

$$
\frac{P_{n}(z)}{[\phi(z)]^{n+1}}=\frac{1}{2 \pi i} \int_{\Gamma} \frac{P_{n}(t) /[\phi(t)]^{n+1}}{t-z} d t
$$

where the integral is taken in the clockwise sense on $\Gamma$. By use of the general inequality

$$
\left|\int f^{1 / p} g^{(p-1) / p} d x\right| \leqq\left(\int|f| d x\right)^{1 / p}\left(\int|g| d x\right)^{(p-1) / p}
$$

setting $f^{1 / p}=P_{n}(t), g^{(p-1) / p}=1 /\left[2 \pi i[\phi(t)]^{n+1}(t-z)\right]$, and by use of the fact that for $t$ on $\Gamma$ we have $|\phi(t)|=1$, it follows from (1) that for $z$ on $C_{R}$ we have

$$
\left|\frac{P_{n}(z)}{[\phi(z)]^{n+1}}\right| \leqq \rho \frac{1}{2 \pi}\left(\int_{\Gamma} \frac{|d t|}{\mid t-z^{p(p-1)}}\right)^{(p-1) / p}
$$

The second factor on the right is uniformly bounded for all $z$ on $C_{R}$; on $C_{R}$ we have by definition $|\phi(z)|=R$; hence inequality (2) follows for $z$ on $C_{R}$; but since this inequality holds for $z$ on $C_{R}$ it also holds for $z$ on or within $C_{R}$, and the lemma is established.

3. Approximation on a Jordan arc. We apply the Lemma in proving

THEOREM II. Let $C$ be an arbitrary rectifiable Jordan arc, and suppose we have for $n=1,2, \cdots$ the inequality

$$
\int_{C}\left|f(z)-\pi_{n}(z)\right|^{p} n(z)|d z| \leqq \frac{M}{R^{n p}}, \quad p, R>1,
$$

where $\pi_{n}(z)$ is a polynomial of degree $n$ and where the function $n(z)$ is continuous and positive on $C$. Then the sequence $\left\{\pi_{n}(z)\right\}$ converges throughout the interior of $C_{R}$, uniformly on any closed point set interior to $C_{R}$.

Let $N$ be a positive number less than $n(z)$ on $C$; we have

$$
\int_{C}\left|f(z)-\pi_{n}(z)\right|^{p}|d z| \leqq \frac{M}{N R^{n p}} .
$$

From the general inequality

$$
\int\left|\chi_{1}+\chi_{2}\right|^{p} d x \leqq 2^{p-1} \int\left|\chi_{1}\right|^{p} d x+2^{p-1} \int\left|\chi_{2}\right|^{p} d x
$$

it follows that 


$$
\begin{aligned}
\int_{C}\left|\pi_{n+1}(z)-\pi_{n}(z)\right|^{p}|d z| & \leqq 2^{p-1} \int_{C}\left|f(z)-\pi_{n}(z)\right|^{p}|d z| \\
+2^{p-1} \int_{C}\left|f(z)-\pi_{n+1}(z)\right|^{p}|d z| & \\
& \leqq \frac{2^{p-1} M}{N R^{n p}}+\frac{2^{p-1} M}{N R^{(n+1) p}}=\frac{2^{p-1} M\left(1+R^{p}\right)}{N R^{(n+1) p}} .
\end{aligned}
$$

This inequality is of form (1), for the polynomial $\pi_{n+1}(z)-\pi_{n}(z)$ is of degree $n+1$. If we choose an arbitrary $R_{1}$ less than $R$, we have by (2) an inequality of the form

$$
\left|\pi_{n+1}(z)-\pi_{n}(z)\right| \leqq M_{1}\left(\frac{R_{1}}{R}\right)^{n+1}, \quad z \text { on or within } C_{R_{1}},
$$

from which it follows that the sequence $\left\{\pi_{n}(z)\right\}$ converges interior to $C_{R}$, uniformly on any closed point set interior to $C_{R}$.

The function $\omega(z)$ which is the limit of the sequence $\left\{\pi_{n}(z)\right\}$ on $C$ naturally coincides with the given function $f(z)$ on $C$ except perhaps on a set of measure zero. For the uniform convergence of the sequence to the function $\omega(z)$ as proved implies

$$
\lim _{n \rightarrow \infty} \int_{C}\left|\omega(z)-\pi_{n}(z)\right|^{p}|d z|=0 .
$$

Let an arbitrary positive $\epsilon$ be given. Choose a particular $n$ so large that we have

$$
\int_{C}\left|f(z)-\pi_{n}(z)\right|^{p}|d z|<\epsilon, \quad \int_{C}\left|\omega(z)-\pi_{n}(z)\right|^{p}|d z|<\epsilon .
$$

It follows from (5) that we have

$$
\int_{C}|f(z)-\omega(z)|^{p}|d z|<2^{p} \epsilon
$$

from which it follows that $f(z)$ and $\omega(z)$ are equal on $C$ except perhaps on a point set of measure zero. If the given function $f(z)$ is analytic on $C$, it must be identical with $\omega(z)$, for the two functions coincide in an infinity of points of $C$.

4. Polynomials of best approximation. There is a sort of converse (not exact) of Theorem II which can be established quite easily: 
THEOREM III. If the function $f(z)$ is analytic on and within $C_{R}$, then there exist polynomials $\pi_{n}(z)$ of respective degrees $n=1,2, \cdots$ such that we have

$$
\int_{C}\left|f(z)-\pi_{n}(z)\right|{ }^{p} n(z)|d z| \leqq \frac{M}{R^{n p}} .
$$

In fact by Theorem I we have

$$
\left|f(z)-\pi_{n}(z)\right| \leqq \frac{M_{1}}{R^{n}}, \quad z \text { on } C,
$$

and this leads directly to (4).

Theorem II applies directly to the polynomial of best approximation to $f(z)$ in the sense of least weighted $p$ th powers measured on $C$. If the integral in the left-hand member of (4) is not greater when formed for the polynomial $\pi_{n}(z)$ than when formed for any other polynomial of degree $n$, then $\pi_{n}(z)$ is called a polynomial of best approximation to $f(z)$ in the sense of least weighted $p$ th powers measured on $C$. This polynomial of best approximation exists and is unique; see $\$ 8$ below.

THEOREM IV. Let $C$ be an arbitrary rectifiable Jordan arc. If the function $f(z)$ is analytic within $C_{R}$, then the sequence of polynomials $\pi_{n}(z)$ of best approximation to $f(z)$ on $C$ in the sense of least weighted pth powers as measured by integration on $C$, converges to $f(z)$ within $C_{R}$, uniformly on any closed point set interior to $C_{R}$.

Let $R_{1}<R$ be arbitrary. Then by Theorem III we have for some sequence of polynomials $\pi_{n}(z)$ inequality (4) with $R$ replaced by $R_{1}$. Since this inequality holds for some sequence of polynomials it necessarily holds for the sequence of polynomials of best approximation, whence Theorem IV follows by Theorem II.

The special case of Theorem IV in which $n(z) \equiv 1, p=2$, and $C$ is a segment of the axis of reals, leads to the expansion of $f(z)$ in a series of Legendre polynomials, and the result in this case is well known.

We have required in the present discussion that $n(z)$ should actually be greater than zero. If that requirement is replaced by the one of demanding that $n(z)$ should be merely greater than or equal to zero, the situation is somewhat altered. Let us suppose for instance that we have $n(z)$ greater than zero on a closed subset $C^{\prime}$ of $C$; let us take the simple case where $C^{\prime}$ consists of a finite number of arcs of $C$. The first part of Theorem I has not been proved* for such sets as $C^{\prime}$, but the second part has been proved (loc.

* The present writer hopes shortly to publish some results in this connection. 
cit.). Thus a more special result than Theorem IV can now be established. We assume the function $f(z)$ to be analytic interior to $C_{R}$. Then the sequence of polynomials $\pi_{n}(z)$ of best approximation to $f(z)$ on $C$, in the sense of least weighted pth powers as measured by integration on $C$, converges to $f(z)$ within $C_{R}{ }^{\prime}$, uniformly on any closed point set interior to $C_{R}{ }^{\prime}$. The point set $C_{R}{ }^{\prime}$ is defined as above, namely the curve or curves $|\phi(z)|=R$, where the function $w=\phi(z)$ maps the exterior of $C^{\prime}$ onto the exterior of $\gamma:|w|=1$ so that the points at infinity correspond to each other. To be sure, this conformal map is not smooth, but the mapping function nevertheless exists and the point set $C_{R}{ }^{\prime}$ is uniquely defined.

The point set $C^{\prime}$ just treated can readily be generalized to include the new case that the function $n(z)$ is positive on the set $C^{\prime}$, consisting of a finite number of intervals of some Jordan $\operatorname{arc} C$, except that the function $n(z)$ may vanish at some or all of the end points of those intervals. We consider a new variable closed point set $C^{\prime \prime}$ consisting of subintervals of the respective intervals of $C$ belonging to $C^{\prime}$, and the point set $C^{\prime \prime}$ varies monotonically and eventually includes any preassigned point of $C^{\prime}$ not an end point of an interval. Then the point set $C_{R}{ }^{\prime \prime}$ liés near to $C_{R}{ }^{\prime}$ and approaches $C_{R}{ }^{\prime}$ uniformly. Hence if the function $f(z)$ is analytic interior to $C_{R}$, the sequence of polynomials $\pi_{n}(z)$ of best approximation to $f(z)$ on $C$, in the sense of least weighted $p$ th powers as measured by integration on $C$, converges to $f(z)$ within $C_{R^{\prime}}$, uniformly on any closed point set interior to $C_{R}{ }^{\prime}$.

The remark just made on the vanishing of the weight function has obvious application to results of the present paper other than Theorem IV, and also to the study of other methods of approximation, notably that of Tchebycheff. In the remainder of the present paper we assume as before that the weight function is actually greater than zero.

The vanishing of the weight function has been studied recently (before the formulation of the remark just made) by Professor Dunham Jackson, for the case that approximation is on a line segment.*

5. More general rectifiable boundaries. In Theorems II-IV we have required that $C$ should be a rectifiable Jordan arc. Inspection of the proof shows, however, that the reasoning is valid in much more general cases, in fact is valid if $C$ is an arbitrary limited point set whose complement is simply connected and which is bounded by a finite number of rectifiable Jordan curves and arcs. Thus in the proof of the Lemma, the Jordan curve $\Gamma$ may consist of the entire boundary of such a point set $C$, and the parts of the 629.

* Abstract published in the Bulletin of the American Mathematical Society, vol. 36 (1930), p. 
boundary of $C$ which are Jordan arcs not bounding Jordan regions belonging to $C$ are counted twice in considering $\Gamma$; the $z$-plane is cut along those Jordan arcs. The reader can easily write the suitable generalizations of Theorems II and III for this case. We state explicitly the generalization of Theorem IV:

THEOREM V. Let $C$ be an arbitrary limited point set whose complement is simply connected and which is bounded by a finite number of rectifiable Jordan curves and arcs. If the function $f(z)$ is analytic within $C_{R}$, the sequence of polynomials $\pi_{n}(z)$ of best approximation to $f(z)$ on $C$, in the sense of least weighted pth powers as measured by integration on the boundary of $C$, converges to $f(z)$ within $C_{R}$, uniformly on any closed point set interior to $C_{R}$.

There is perhaps some doubt as to whether, in the integral which is the measure of the approximation, the single Jordan arcs of $C$ should be counted singly or doubly. The reader will easily see that this is a matter of complete indifference. Moreover, even an infinite number of Jordan curves and arcs are allowable in Theorem $\mathrm{V}$ if the sum of their lengths is finite.

Theorem $\mathrm{V}$ includes not merely Theorem IV but also the interesting case where $C$ is a region bounded by a single rectifiable Jordan curve. ${ }^{*}$ In the study of such a region, one may also approximate to an arbitrary function analytic in the interior, continuous in the closed region. An easy corollary $\dagger$ of results obtainable in this way is

THEOREM VI. Let $C$ be an arbitrary limited point set whose complement is simply connected and which is bounded by a finite number of rectifiable Jordan curves and arcs. If the function $f(z)$ is continuous on $C$, analytic in the interior points of $C$, then the sequence of polynomials of best approximation to $f(z)$ on $C$, in the sense of least weighted pth powers as measured by integration on the boundary of $C$, converges to $f(z)$ in the interior points of $C$, uniformly on any closed point set composed entirely of interior points of $C$.

6. Approximation on more general point sets: a lemma. If we study approximation to an arbitrary function $f(z)$ on a non-rectifiable Jordan arc $C$, the measure of approximation used in Theorems II-V may have no meaning. We shall now study the related measure of approximation after the mapping of $D$ (defined as in \$1) onto the exterior of the circle $\gamma:|w|=1$ so that the points at infinity correspond to each other. That is, the new measure of approximation is an integral over $\gamma$ instead of over the boundary of $C$. This enables us to consider point sets $C$ much more general than Jordan arcs, but nevertheless

* See Walsh, loc. cit., Theorem III.

$\dagger$ Loc. cit., Theorem IX, and also Walsh, these Transactions, vol. 30 (1928), pp. 472-482, Theorem IX. 
the results already established are not included in those about to be taken up. Julia has recently* used conformal mapping in connection with the measure of approximation of harmonic functions by harmonic polynomials, but he restricts $C$ to being a Jordan region and maps the interior of $C$ onto the interior of $\gamma$, which is less favorable for our present purposes than the mapping of the exterior as we study it here.

Lemma. Let $C$ be an arbitrary limited point set whose complement $D$ is simply connected. If we have

$$
\int_{\gamma}\left|P_{n}(z)\right| p|d w| \leqq \rho^{p}, p>1,
$$

where $P_{n}(z)$ is a polynomial in $z$ of degree $n$, then we have also

$$
\left|P_{n}(z)\right| \leqq M_{R} \rho R^{n+1},
$$

$z$ on or within $C_{R}$, where $M_{R}$ depends on $R$ but not on $n$ nor. $\rho$.

Under the conformal map $w=\phi(z), z=\psi(w)$, of $D$ onto the exterior of $\gamma$ so that the points at infinity correspond to each other, $\lim _{r \rightarrow 1, r>1} \psi\left(r e^{i \phi}\right)$ exists $\dagger$ for almost all values of $\phi$. Thus

$$
\lim _{r \rightarrow 1, r>1} P_{n}\left[\psi\left(r e^{i \phi}\right)\right], \quad \phi \text { constant }
$$

likewise exists for almost all values of $\phi$, and these are the values of $P_{n}(z)$ on $\gamma$ which we contemplate in (6). The function $P_{n}(z) / w^{n+1}$ is an analytic function of $w$ exterior to $\gamma$ and approaches zero when $w$ becomes infinite. Thus we have, for an arbitrary point $w$ exterior to $\gamma$,

$$
\frac{P_{n}(z)}{w^{n+1}}=\frac{1}{2 \pi i} \int_{\gamma} \frac{P_{n}[\psi(t)] / t^{n+1}}{t-w} d t,
$$

where the integral is taken over the circle $\gamma$ in the clockwise sense. Equation (8) is proved by considering first the equation corresponding to (8) where the integral is taken over a circle $|w|=1+1 / m$ and then by taking the limit as $m$ becomes infinite. If $w$ is fixed exterior to $\gamma$, the integrand approaches a limit on almost every ray through the origin, and the integrand is uniformly bounded independently of $m$, so the limiting process $(m \rightarrow \infty)$ is justified.

The method of proof used in $\$ 2$ now yields

$$
\left|\frac{P_{n}(z)}{w^{n+1}}\right| \leqq M_{R} \cdot \rho, \quad \text { for all }|w|=R,
$$

\footnotetext{
* Acta Litterarum ac Scientiarum (Szeged), vol. 4 (1929), pp. 217-226.

$\dagger$ Fatou's theorem applies directly to the function $\psi(w) / w$.
} 
where $M_{R}$ depends only on $C$ and $R$. Transformation back to the $z$-plane now gives (9) for all $z$ on $C_{R}$ and hence yields (7) for all $z$ on or within $C_{R}$.

The analogue of Theorem II follows by the proof used in that theorem; the function $n(w)$ is supposed positive and continuous on $\gamma$ :

THEOREM VII. Let $C$ be an arbitrary limited point set whose complement is simply connected. Then the relation

$$
\int_{\gamma}\left|f(z)-\pi_{n}(z)\right|{ }^{p} n(w)|d w| \leqq \frac{M}{R^{n p}}, \quad p>1,
$$

where $\pi_{n}(z)$ is a polynomial of degree $n$, implies the convergence of the sequence $\left\{\pi_{n}(z)\right\}$ throughout the interior of $C_{R}$, uniformly on any closed point set interior to $C_{R}$.

The values of the polynomials $\pi_{n}(z)$ on $\gamma$ considered in (10) are of course the values considered as in the lemma; we make the definition

$$
\pi_{n}\left[\psi\left(e^{i \phi}\right)\right]=\lim _{r \rightarrow 1, r>1} \pi_{n}\left[\psi\left(r e^{i \phi}\right)\right], \phi \text { constant }
$$

If the given function $f(z)$ is known to be analytic and bounded in the neighborhood of the boundary of $D$, a similar definition may be used for $f(z)$ on $\gamma$. No matter how $f(z)$ may be given on $\gamma$ originally, that function can differ on $\gamma$ from the function $F(z)=\lim _{n \rightarrow \infty} \pi_{n}\left[\psi\left(e^{i \phi}\right)\right]$ at most on a set of measure zero. For the sequence $\left\{\pi_{n}(z)\right\}$ converges uniformly on any closed point set interior to $C_{R}$, hence converges uniformly in the neighborhood of the boundary of $C$, and converges uniformly on $C$ in the point set $z=\psi\left(e^{i \phi}\right)$ corresponding to almost all points of $\gamma$. On this point set of $\gamma$ (i.e. for which $\lim _{r \rightarrow 1, r>1} \psi\left(r e^{i \phi}\right)$ exists), the sequence $\pi_{n}\left[\psi\left(e^{i \phi}\right)\right]$ converges uniformly, hence, by the reasoning used in $\S 3$, the functions $F(z)$ and $f(z)$ differ at most on a set of $\gamma$ of measure zero. If $f(z)$ is given analytic on $C$, the two functions $f(z)$ and $F(z)$ are identical.

7. Convergence of the sequehce of polynomials of best approximation. Results analogous to Theorems III and IV can now be establisked, by methods similar to those previously used.

THEOREM VIII. If the function $f(z)$ is analytic on and within $C_{R}$, where $C$ is an arbitrary closed limited point set whose complement $D$ is simply connected, then there exist polynomials $\pi_{n}(z)$ of respective degrees $n$ and a number $M$ such that

$$
\int_{\gamma}\left|f(z)-\pi_{n}(z)\right|{ }^{p} n(w)|d w| \leqq \frac{M}{R^{n p}}, \quad p>1
$$


here the correspondence between $z$ and $w$ is found by mapping $D$ onto the exterior of $\gamma:|w|=1$ so that the points at infinity correspond to each other.

To be sure, this conformal map is strictly defined merely in the open regions $D$ and $|w|>1$ respectively, but we consider as before the map to be defined in the closed regions by continuity whenever this is possible. This extension of the original definition of the map is sufficient to define $f(z)$ and $\pi_{n}(z)$ on $\gamma$ and to ensure the validity of (10).

By Theorem I there exist polynomials $\pi_{n}(z)$ of respective degrees $n$ such that we have for $z$ on $C$

$$
\left|f(z)-\pi_{n}(z)\right| \leqq \frac{M_{1}}{R^{n}} .
$$

Under the conformal map $w=\phi(z), z=\psi(w)$, the limit

$$
\lim _{r \rightarrow 1, r>1} \psi\left(r e^{i \phi}\right)
$$

exists for almost all values of $\phi$ and is denoted as before by $\psi\left(e^{i \phi}\right)$. For these same values of $\phi$, the limits

$$
\lim _{r \rightarrow 1, r>1} f\left[\psi\left(r e^{i \phi}\right)\right], \lim _{r \rightarrow 1, r>1} \pi_{n}\left[\psi\left(r e^{i \phi}\right)\right]
$$

exist and are equal respectively to

$$
f\left[\psi\left(e^{i \phi}\right)\right], \pi_{n}\left[\psi\left(e^{i \phi}\right)\right] .
$$

Thus inequality (11) obtains for these same values of $\phi$, that is, for almost all values of $\phi$, and (10) follows at once.

If the integral in the left-hand member of (10) is not greater when formed for a particular polynomial $\pi_{n}(z)$ of degree $n$ than when formed for any other polynomial $\pi_{n}(z)$ of degree $n$, that polynomial is called a polynomial of best approximation to $f(z)$ on $C$, in the sense of least weighted pth powers as measured on $\gamma$. This polynomial of best approximation exists and is unique; see $\S 8$ below.

Theorem IX. Let $C$ be an arbitrary closed limited point set whose complement $D$ is simply connected. If the function $f(z)$ is analytic interior to $C_{R}$, then the sequence of polynomials $\pi_{n}(z)$ of best approximation to $f(z)$ on $C$ in the sense of least weighted pth powers as measured by integration on $\gamma:|w|=1$ after conformal mapping of D onto the exterior of $\gamma$, converges to $f(z)$ within $C_{R}$, uniformly on any closed point set interior to $C_{R}$.

The proof of Theorem IX follows directly the proof of Theorem IV, by application of Theorems VII and VIII instead of II and III, and is left to the reader. 
So far as the writer is aware, the point set $C$ of Theorem IX is the most general one that has been considered in the literature in connection with polynomials belonging to a region or to a point set. For in the case $n(z) \equiv 1$, $p=2$, the polynomials $\pi_{n}(z)$ are of the form

$$
\pi_{n}(z)=a_{1} p_{1}(z)+a_{2} p_{2}(z)+\cdots+a_{n} p_{n}(z),
$$

where the polynomials $p_{i}(z)$ depend only on $C$ and not on $f(z)$ nor on $n$, and where the coefficients $a_{i}$ depend on $f(z)$ but not (for $n \geqq i$ ) on $n$.

We remark too that all the results of the present paper hold for the case $p=1$, and that many of the results are valid provided merely $p>0$. The requisite modifications in the proofs and in the formulations of the theorems are left to the reader.

8. Existence and uniqueness of polynomials of best approximation. It is our purpose now to prove

Theorem X. In Theorems VI and IX, the polynomial $\pi_{n}(z)$ of best approximation exists and is unique.

Let us give the proof for the polynomial of best approximation in Theorem IX; the case of Theorem VI is in reality somewhat simpler, and the requisite modifications can be made by the reader.

The measure of approximation

$$
\epsilon_{n}^{(i)}=\int_{\gamma}\left|f(z)-\pi_{n}^{(i)}(z)\right|^{p} n(w)|d w|
$$

is positive or zero, so there exists a greatest lower bound $\epsilon$ of the set of all numbers $\epsilon_{n}^{(i)}$ corresponding to polynomials $\pi_{n}^{(i)}(z)$ of degree $n$. There exists a sequence of numbers $\epsilon_{n}^{(1)}, \epsilon_{n}^{(2)}, \cdots$ (all belonging to the set just noted) which approaches $\epsilon$, so for the corresponding polynomials $\pi_{n}^{(i)}(z)$ we have

$$
\lim _{i \rightarrow \infty} \int_{\gamma}\left|f(z)-\pi_{n}^{(i)}(z)\right|^{p} n(w)|d w|=\epsilon .
$$

By means of (5) we can write

$$
\begin{aligned}
\int_{\gamma}\left|\pi_{n}^{(i)}(z)\right| p|d w| \leqq & 2^{p-1} \int_{\gamma}|f(z)|^{p}|d w| \\
& +2^{p-1} \int_{\gamma}\left|f(z)-\pi_{n}^{(i)}(z)\right|^{p}|d w| \leqq M,
\end{aligned}
$$

where $M$ is suitably chosen. 
The lemma of $\S 6$ now informs us that the sequence of polynomials $\left\{\pi_{n}^{(i)}(z)\right\}, i=1,2, \cdots$, is uniformly bounded in $C_{R}$, where $R$ is arbitrary. Hence this family of functions is normal and from it can be extracted a subsequence converging uniformly in an arbitrary closed subregion of $C_{R}$, say in $C_{R_{1}}, R_{1}<R$. In particular this subsequence converges uniformly on the boundary of $C$ and hence on $\gamma$, or at least almost everywhere on $\gamma$, in the points of $\gamma$ which correspond to the values of $\phi$ for which

$$
\lim _{r \rightarrow 1, r>1} \psi\left(r e^{i \phi}\right), \quad \phi \text { constant, }
$$

exists. The analytic function which is the limit of the subsequence is naturally a polynomial $\pi_{n}(z)$ of degree $n$; this follows as an easy application of Lagrange's interpolation formula. ${ }^{*}$ The uniformity of the convergence yields at once from (12)

$$
\int_{\gamma}\left|f(z)-\pi_{n}(z)\right|{ }^{p} n(w)|d w|=\epsilon,
$$

and this is the proof of the existence of the polynomial of best approximation.

The uniqueness of the polynomial of best approximation can be established by known methods. $\dagger$ It will be noticed, however, that Theorems VI and IX are entirely independent of this uniqueness.

9. Exact region of uniform convergence of sequences of polynomials of best approximation. We shall now prove the following theorem:

TheOREM XI. Let the function $f(z)$ be analytic on the point set $C$ and interior to $C_{R}$ but have a singularity on $C_{R}$. Then any sequence of polynomials $\pi_{n}(z)$ (of respective degrees $n$ ) which converges on $C$ to $f(z)$ with the same degree of approximation as the sequence of polynomials of best approximation, converges to $f(z)$ interior to $C_{R}$, uniformly on any closed point set interior to $C_{R}$, and converges uniformly in no region which contains in its interior an arc of $C_{R}$.

In this theorem the term "best approximation" may be taken in any of the senses we have previously considered: (1) in the sense of Tchebycheff, $C$ being an arbitrary limited closed point set whose complement is simply connected; (2) in the sense of least weighted $p$ th powers $(p \geqq 1)$ measured by integration over the boundary of the limited region $C$, this boundary being an arbitrary rectifiable Jordan curve; (3) in the sense of least weighted $p$ th powers on the circumference $\gamma:|w|=1$, where $C$ is an arbitrary limited sim-

\footnotetext{
* See for instance de la Vallée Poussin, Approximation des Fonctions, Paris, 1919, §55.

$\dagger$ See for instance Julia, loc. cit., pp. 221-222.
} 
ply connected region and is mapped conformally onto the interior of $\gamma ;(4)$ in the sense of least weighted $p$ th powers as measured by integration over the area of $C$, the region $C$ being an arbitrary limited region; (5) in the sense of least weighted $p$ th powers as measured by integration over $C$, the point set $C$ being an arbitrary rectifiable Jordan arc; (6) in the sense of least weighted $p$ th powers as measured by integration on the circumference $\gamma:|w|=1$, where $C$ is an arbitrary limited closed point set whose complement $D$ is simply connected and $D$ is mapped onto the exterior of $\gamma$ so that the points at infinity correspond to each other.

Only the last clause in the statement of Theorem XI contains anything new. The proofs in all of the cases (1)-(6) are so similar that it is not necessary to give them all in detail. Let. us restrict ourselves to a typical case, namely (5), where the point set $C$ is a rectifiable Jordan arc.

The requirement that the sequence $\left\{\pi_{n}(z)\right\}$ should converge to $f(z)$ with the same degree of approximation as the sequence of polynomials of best approximation is taken to mean that for an arbitrary $R_{1}<R$ there exists $M$ such that we have

$$
\int_{C}\left|f(z)-\pi_{n}(z)\right|^{p} n(z)|d z| \leqq \frac{M}{R_{1}^{n p}} .
$$

A consequence of this inequality (compare the proof of Theorem II) is that for an arbitrary $R_{2}<R_{1}<R$ there exists $M^{\prime}$ such that we have

$$
\left|f(z)-\pi_{n}(z)\right| \leqq M^{\prime}\left(\frac{R_{2}}{R_{1}}\right)^{n}, \quad z \text { on or within } C_{R_{2}} .
$$

Inequality (14) asserts that the sequence $\left\{\pi_{n}(z)\right\}$ converges on or within $C_{R_{2}}$ like a convergent geometric series. It follows from a very general theorem due to Ostrowski* that in any closed region interior to the region of uniform convergence of the given sequence we also have convergence like a convergent geometric series. If Theorem XI is not true, the sequence $\pi_{n}(z)$ converges in some region $D^{\prime \prime}$ which contains points interior to $C_{R}$-and on any closed point set interior to $C_{R}$ inequality (14) holds for proper choice of $R_{1}$ and $R_{2}$-and such that $D^{\prime \prime}$ contains also some closed region $D^{\prime}$ which lies exterior to $C_{R}$. Let us take the convergence in $D^{\prime}$ in the form

$$
\left|f(z)-\pi_{n}(z)\right| \leqq \frac{N}{S^{n}}, \quad S>1, \quad z \text { in } D^{\prime} .
$$

* Abhandlungen aus dem Mathematischen Seminar der Hamburgischen Universität, vol. 1 (1922), pp. 327-350; p. 329. 
It is no loss of generality here to take $S$ less than $R$. We set $R_{2}=R / S$, so we have $1<R_{2}<R_{1}<R$, with $R / R_{2}=S>R_{1} / R_{2}=S_{1}$. Then by (14) and (15) we can write

$$
\left|f(z)-\pi_{n}(z)\right| \leqq \frac{N^{\prime}}{S_{1}{ }^{n}}, z \text { on or within } C_{R_{2}} \text {, or } z \text { in } D^{\prime}
$$

it will be noted that $C_{R_{2}}$ and $D^{\prime}$ lie exterior to each other.

We are now in a position to apply a generalization of the second part of Theorem I, the present hypothesis being that an inequality of type (16) is given not merely in a single region but in two disconnected regions. Let $u(x, y)$ be Green's function for the region $E$, the exterior of $C_{R_{2}}$ and $D^{\prime}$ : the function $u(x, y)$ is harmonic in $E$, continuous in the corresponding closed region except at infinity, zero on the boundary of $E$, and can be written at infinity as $\log \left(x^{2}+y^{2}\right)^{1 / 2}$ plus a function harmonic at infinity. Then it follows* that the sequence $\left\{\pi_{n}(z)\right\}$ converges in the interior $I_{S_{1}}$ of the locus $u(x, y)=\log S_{1}$, and the sequence converges uniformly on any closed point set interior to the locus. This locus may consist of a single curve or of two curves, and the set $I_{S_{1}}$ is the interior of the one or of both, but in the latter case we shall be particularly concerned with $I_{S_{1}^{\prime}}^{\prime}$, that one of the two curves which contains $C_{R_{2}}$ in its interior. Consider also the locus $I_{S_{1}^{\prime \prime}}: u_{1}(x, y)=\log$ $S_{1}$, where $u_{1}(x, y)$ is harmonic exterior to $C_{R_{2}}$, continuous in the corresponding closed region except at infinity, zero on $C_{R_{2}}$, and can be expressed at infinity as $\log \left(x^{2}+y^{2}\right)^{1 / 2}$ plus a function harmonic at infinity. The curve $I_{S_{1}^{\prime \prime}}$ is interior to the curve $I_{S_{1}^{\prime}}^{\prime}$. Indeed on the boundary of $E$ we have

$$
u(x, y)-u_{1}(x, y) \leqq 0 ;
$$

the left-hand member is harmonic in $E$ even at infinity, so interior to $E$ the strong inequality obtains:

$$
u(x, y)-u_{1}(x, y)<0 .
$$

That is, on $I_{S_{1}^{\prime \prime}}: u_{1}(x, y)=\log S_{1}$ we have $u(x, y)<\log S_{1}$, so the curve $I_{S_{1}^{\prime \prime}}$ lies interior to $I_{S_{1}^{\prime}}$.

The curve $I_{S_{1}^{\prime}}{ }^{\prime \prime}$ is of course a curve of the family $C_{\rho}$; in fact if the function $w=\phi(z)$ maps the exterior of $C$ onto the exterior of $\gamma$, the function $w=\phi(z) / R_{2}$ maps the exterior of $C_{R_{2}}$ onto the exterior of $\gamma$, and $I_{S_{1}^{\prime \prime}}$ is defined as the curve $|\phi(z)| / R_{2}=S_{1}$. The curve $C_{R}$ is the curve of the family $I_{S_{1}^{\prime \prime}}$ corresponding to $|\phi(z)| / R_{2}=R / R_{2}$, that is, the curve $C_{R}$ is the curve $I_{S}^{\prime \prime}$, and lies interior to the curve $I_{S^{\prime}}$. Allow now $R_{1}$ to approach $R, R_{2}$ being fixed, so that $S_{1}$ ap-

\footnotetext{
* See p. 229 of the reference given for Theorem I.
} 
proaches $S$ and the point sets $I_{S_{1}}, I_{S_{1}}^{\prime}, I_{S_{1}}^{\prime \prime}$ are variable, approaching uniformly* the respective point sets $I_{S}, I_{S}{ }^{\prime}, I_{S^{\prime \prime}}{ }^{\prime}$. The sequence $\left\{\pi_{n}(z)\right\}$ always converges uniformly on an arbitrary closed point set interior to $I_{S_{1}}$, hence converges uniformly on an arbitrary closed point set interior to $I_{S}$. The curve $C_{R}$ lies interior to $I_{S}$, so the sequence $\left\{\pi_{n}(z)\right\}$ converges uniformly on a closed point set containing $C_{R}$ in its interior, yet the limit of the sequence is $f(z)$ in $C$, hence also in $I_{S}$, and $f(z)$ has a singularity on $C_{R}$. This contradiction completes the proof.

Theorem XI has already been established by various writers and by other methods, and even more specific results, for certain special types of approximation (1)-(6). References to the literature have already been given for these cases.

Many consequences (e.g. Lückensätze) other than Theorem XI follow from the results of Ostrowski (loc. cit.) as applied to the present sequence $\left\{\pi_{n}(z)\right\}$ in the cases (1)-(6). We mention the theorem analogous to that of Jentzsch, that each point of $C_{R}$ is a limit point of the zeros of polynomials of the sequence $\left\{\pi_{n}(z)\right\}$.

Theorem XI has obvious application to results on overconvergence recently proved $\dagger$ concerning approximation to rational functions, or to arbitrary analytic functions by polynomials satisfying prescribed auxiliary conditions. In each case (loc. cit., Theorems A, B, 4, 5, 6, 7, 9, 12, 15; \$12.2) the sequence of approximating polynomials converges to its limit with the same degree of approximation as does the sequence of polynomials of best approximation. Hence in each case there is no region of uniform convergence containing in its interior any arc of the curve $C_{R}$, provided that we have uniform convergence interior to $C_{R_{1}}$ whenever $R_{1}$ is less than $R$, and that the limit function has a singularity on $C_{R}$.

It is not without interest to notice that under the hypothesis of Theorem $\mathrm{XI}$, divergence of the sequence of polynomials $\left\{\pi_{n}(z)\right\}$ at all points exterior to $C_{R}$ cannot be proved. Indeed, it can be shown (see the reference just given, $\$ 12.2)$ that if the function $f(z)$ satisfies the hypothesis of Theorem XI, and if there are assigned arbitrary points $\beta_{i}$, finite in number and exterior to $C_{R}$, then there exist polynomials $\pi_{n}(z)$ which approximate $f(z)$ on $C$ with the same degree of approximation as the sequence of polynomials of best approximation $\ddagger$ and which for $n$ sufficiently large satisfy the auxiliary conditions

* See for instance Lebesgue, Palermo Rendiconti, vol. 24 (1907), pp. 371-402.

† Walsh, these Transactions, vol. 32 (1930), pp. 335-390.

$\ddagger$ This fact is there proved merely for approximation measured in the sense of Tchebycheff, but that implies the corresponding fact for any of the methods (1)-(6) of measuring approximation. 
$\pi_{n}\left(\beta_{i}\right)=0$. This sequence $\left\{\pi_{n}(z)\right\}$ naturally converges not merely interior to $C_{R}$ but also at the points $\beta_{i}$ exterior to $C_{R}$.

The proof of Theorem XI that we have given contains incidentally a proof of the following theorem: If $f(z)$ is analytic interior to $C_{R}$ but has a singularity on $C_{R}$, then neither the sequence of polynomials $\left\{\pi_{n}(z)\right\}$ of best approximation to $f(z)$ on $C$ (measured in any one of the ways considered) nor any other sequence of polynomials which converges on $C$ like the sequence of polynomials of best approximation converges like a geometric series in any region or on any Jordan arc exterior to $C_{R}$.

The result analogous to Theorem XI holds also for the sequences $\left\{\pi_{n}(x\right.$, $y)$ \} of harmonic polynomials of best approximation to harmonic functions, where best approximation is taken in any of the senses (1), (2), (3), (4).* If we assume that this result is false, the sequence $\left\{\pi_{n}(x, y)\right\}$ converges in some region $D^{\prime \prime}$ which contains in its interior an arc of $C_{R}$ and hence $D^{\prime \prime}$ contains some simply connected closed region $D^{\prime}$ which lies exterior to $C_{R}$. The sequence of polynomials $p_{n}(z)$ which vanish at a particular point of $C$ and of which the polynomials $\pi_{n}(x, y)$ are the respective real parts, converges like a convergent geometric series on any closed point set interior to $C_{R}$, $†$ hence converges like a convergent geometric series also in the region $D^{\prime}$. Detailed study of the ratios of these geometric series, as in Theorem XI, yields directly a contradiction.

10. Addendum. $f$ Discussion of case $0<p<1$. In the study of approximation by polynomials to an arbitrary analytic function of a complex variable where approximation is in the sense of least $p$ th powers and is measured by an integral, we have consistently set aside the case $0<p<1$, for inequality (3) is not valid in this case. The case $p>1$ is naturally the most interesting, for here a polynomial of degree $n$ of best approximation always exists and is unique, and $p>1$ includes also the case $p=2$ for which the approximating polynomials have a particularly simple form; the approximating functions may be found by orthogonalization and normalization of the sequence $\left\{[n(z)]^{1 / 2} z^{n}\right\}$. Nevertheless the case $0<p<1$ possesses some interest; in fact Professor Dunham Jackson has recently published§ some results on overconvergence, which are broadly speaking less general than those of the present writer, but which nevertheless treat the cases $0<p<1, p=1$, and $p>1$ without distinction by a single method. We shall therefore now show how the

* The point sets $C$ are restricted as in our previous discussion (loc. cit.) of these measures of approximation for harmonic functions by harmonic polynomials.

† Compare Walsh, Bulletin of the American Mathematical Society, vol. 33 (1927), pp. 591-598.

$\ddagger$ Received by the editors, February 3, 1931.

\&ulletin of the American Mathematical Society, vol. 36 (1930), pp. 851-857. 
methods of the present paper and those previously used by the present writer can be modified so as to include the case $0<p<1$ as well as the case $p \geqq 1$. We shall not take up in detail each of the cases (1)-(6) mentioned in the preceding section, for the treatments in all of these cases are similar; we do take up (2) and (4) respectively in detail.

Lemma. If each of the functions $f_{n}(z), n=1,2, \cdots$, is analytic and uniformly bounded ${ }^{*}$ interior to the rectifiable Jordan curve $C$, and if we set

$$
\int_{C}\left|f_{n}(z)\right|^{p} \cdot|d z|=\epsilon_{n}, \quad p>0,
$$

then $\epsilon_{n} \rightarrow 0$ implies $f_{n}(z) \rightarrow 0$ for $z$ interior to $C$, uniformly on any closed point set $C^{\prime}$ interior to $C$. Moreover, we have

$$
\left|f_{n}(z)\right| \leqq Q \epsilon_{n}{ }^{1 / p}, \quad z \text { on } C^{\prime},
$$

where $Q$ depends on $C^{\prime}$ and on $p$ but not on $f_{n}(z)$.

The boundary value of $f_{n}(z)$ exists almost everywhere on $C$, for approach to an arbitrary point of $C$ along the normal, and it is this boundary value that is used in (17).

Let the zeros of $f_{n}(z)$, if any, interior to $C$ be $\alpha_{1}, \alpha_{2}, \cdots$. We omit from the given sequence $f_{n}(z)$ any functions which vanish identically interior to $C$; it is sufficient to prove the Lemma for the remaining sequence. Consider the function

$$
F_{n}(z)=\frac{\left|f_{n}(z) \prod\right| \phi\left(\alpha_{i}\right) \mid}{\prod \frac{\phi(z)-\phi\left(\alpha_{i}\right)}{\phi(z)-1 / \bar{\phi}\left(\alpha_{i}\right)}}
$$

where $w=\phi(z)$ is a function which maps the interior of $C$ conformally on to the interior of the unit circle $|w|=1$. There may be an infinity of points $\alpha_{i}$, but if so the infinite products in the right-hand member of (19) converge, by Blaschke's theorem. We assume $\phi\left(\alpha_{i}\right) \neq 0$, which involves no loss of generality. The function $F_{n}(z)$ is analytic and has no zeros interior to $C$, and has the same modulus as $f_{n}(z)$ on $C$. The function $\left(F_{n}(z)\right)^{p}$ is likewise analytic and uniformly bounded interior to $C$, if we consider an arbitrary determination of the $p$ th power at a point interior to $C$ and its analytic extension, so we have Cauchy's integral

* The requirement of uniform boundedness is inserted here for simplicity. It can, in fact, be replaced by the mere existence of the integrals in (17), where the boundary values of $f_{n}(z)$ on $C$ are the limit values obtained almost everywhere on $C$ by normal approach, together with the validity of Cauchy's integral formula. 


$$
\left[F_{n}(z)\right]^{p}=\frac{1}{2 \pi i} \int_{C}\left[F_{n}(t)\right]^{p} \frac{d t}{t-z}
$$

Cauchy's integral is naturally valid here, for the boundary values of $\left(F_{n}(z)\right)^{p}$ for normal approach to $C$ exist almost everywhere on $C$. If $l$ denotes the length of $C$ and $\delta$ denotes the minimum distance from $C^{\prime}$ to $C$, we have

$$
\left|F_{n}(z)\right|^{p} \leqq \frac{l}{2 \pi \delta} \int_{C}\left|F_{n}(t)\right|^{p}|d t|=\frac{l \epsilon_{n}}{2 \pi \delta}, \quad z \text { on } C^{\prime} .
$$

Each function

$$
\frac{\left|\phi\left(\alpha_{i}\right)\right|}{\frac{\phi(z)-\phi\left(\alpha_{i}\right)}{\phi(z)-1 / \bar{\phi}\left(\alpha_{i}\right)}}
$$

is of absolute value greater than unity for $z$ interior to $C$, so we have from (19)

$$
\left|f_{n}(z)\right|^{p} \leqq\left|F_{n}(z)\right|^{p}, \quad z \text { interior to } C .
$$

The entire lemma, including (18), now follows from (20).

The lemma is of precisely the form for application in the proof of overconvergence in case (2). The lemma also yields the proof of ordinary convergence (not overconvergence) for approximation by polynomials to a function analytic interior to $C$, continuous in the corresponding closed region, in the sense of least weighted $p$ th powers ( $p$ positive, but greater than, equal to, or less than unity) as measured by a line integral over $C$. The proof of this ordinary convergence in case (3) and of overconvergence in cases (3), (5), (6) follows now with only obvious modifications of the methods already given. We need to consider the case (4) more in detail.

LеммA. Let $C$ be an arbitrary region. If each of the functions $f_{n}(z), n=1$, $2, \cdots$, is analytic interior to $C$, and if we set

$$
\iint_{C}\left|f_{n}(z)\right|^{p} d S=\epsilon_{n}, \quad p>0
$$

then $\epsilon_{n} \rightarrow 0$ implies $f_{n}(z) \rightarrow 0$ for $z$ interior to $C$, uniformly on any closed point set $C^{\prime}$ interior to $C$. Moreover, we have

$$
\left|f_{n}(z)\right| \leqq Q \epsilon_{n}{ }^{1 / p}, z \text { on } C^{\prime}
$$

where $Q$ depends on $C^{\prime}$ and on $p$ but not on $f_{n}(z)$. 
The integral

$$
\frac{1}{2 \pi} \int_{0}^{2 \pi}\left|f\left(z_{0}+r e^{i \theta}\right)\right|^{p} d \theta, \quad p>0,
$$

is well known to be a non-decreasing function of $r$, in an arbitrary circle $K$ which together with its interior lies interior to $C$. Here $(r, \theta)$ are polar coordinates with pole at the point $z_{0}$. The limit of this integral as $r$ approaches zero is obviously $\left|f\left(z_{0}\right)\right|^{p}$, from which follows the inequality

$$
\left|f\left(z_{0}\right)\right|^{p} \leqq \frac{1}{2 \pi} \int_{0}^{2 \pi}\left|f\left(z_{0}+r e^{i \theta}\right)\right|^{p} d \theta
$$

We multiply both sides of this inequality by $r d r$ and integrate from zero to $\kappa$, the radius of $K$. The resulting inequality is

$$
\frac{\kappa^{2}}{2}\left|f\left(z_{0}\right)\right|^{p} \leqq \frac{1}{2 \pi} \iint_{K}|f(z)|^{p} d S,
$$

so we may write

$$
\left|f\left(z_{0}\right)\right|^{p} \leqq \frac{1}{\pi \kappa^{2}} \iint_{K}|f(z)|^{p} d S \leqq \frac{\epsilon_{n}}{\pi \kappa^{2}} .
$$

This inequality holds for every point $z_{0}$ interior to $C$ provided merely that the distance from $z_{0}$ to the nearest point of the boundary of $C$ is not less than $\kappa$. The inequality therefore holds for proper choice of $\kappa$ for $z_{0}$ on the boundary of an arbitrary closed point set $C^{\prime}$ interior to $C$ and implies (22) immediately for $z$ on the boundary of $C^{\prime}$. Such an inequality, holding on the boundary of a closed point set $C^{\prime}$, holds at every point of the set, and the lemma is completely established.

This lemma yields the proof of overconvergence in case (4) and also the proof of ordinary convergence for approximation by polynomials to a function analytic interior to a simply connected region $C$, continuous in the corresponding closed region, in the sense of least weighted $p$ th powers $(p>0)$ as measured by a surface integral over $C$, provided that the function can be uniformly approximated as closely as desired in the closed region by polynomials. There are only obvious modifications to be made in the proofs already given.

HaRVARd UNIVERSITY, Cambridge, Mass. 